\title{
Least-Squares Method for Incompressible Two-phase Flow with Large Density and Viscosity Differences
}

\author{
Keunsoo Park, Maria Fernandino, Carlos A. Dorao \\ Norwegian University of Science and Technology \\ Department of Energy and Process Engineering, NTNU, N-7491 Trondheim, Norway \\ keunsoo.park@ntnu.no; maria.fernandino@ntnu.no; carlos.dorao@ntnu.no.
}

\section{Extended Abstract}

Herein, we consider isothermal, incompressible flow of two immiscible fluids with different densities and viscosities. Numerical simulation of the phase field model for such fluids has become popular and important in the last decade. A frequently used model is based on the Cahn-Hilliard system coupled with the Navier-Stokes equation, and broadly there are two branches of this model depending on the definition of mean velocity of two fluids. Lowengrub et al. [1] suggested a thermodynamically consistent model by introducing a mass-averaged velocity. However, with this definition the velocity field is non-solenoidal, and it leads to a considerable difficulty in numerical computation due to complicated coupling between the Cahn-Hilliard and Navier-Stokes equations. On the other hand, by adopting the definition of volume-averaged velocity with the assumption of incompressibility, the velocity field can be divergence-free. Several models with the solenoidal velocity field are proposed with different expression for the effect of density and viscosity differences (see for example [2-5]), and corresponding discretization schemes are also presented. The advection term in the Cahn-Hilliard equation is discretized by the finite element method with Muramn and Resetarinera schemes in [2] and by an upwinding finite volume scheme in [3]. Shen et al. [4] compared the formulations with the gauge-Uzawa scheme and the pressurestabilization scheme for large density ratio.

The objective of this study is to present the numerical scheme with the least-squares spectral element method (LSSEM) to solve such phase field model. As oppose to other numerical methods, the least squares method does not require any special treatment for handling the convection term in the Cahn-Hilliard and Navier-Stokes equations. The fourth-order CahnHilliard equation can be split into two second-order partial differential equations by introducing the chemical potential as an auxiliary variable, and in this study the global differentiability of the set of these two equations is satisfied by approximating the solution with $\mathrm{C}^{1}$ Hermite polynomial functions in each element. A space-time coupled formulation with a time-stepping procedure is adopted for stability and accuracy of the solution in time. Parallelization of the code is necessary to describe the complicated topological changes on the interface onto sufficiently refined grids, and the system is solved by an element-byelement conjugate gradient method.

The falling droplet example is addressed to point out the role played by the density and viscosity ratio of the two fluids. Three quantities are selected to investigate the effects of density ratio $\lambda_{\rho}=\rho_{\text {vap }} / \rho_{\text {liq }}$ and viscosity ratio $\lambda_{\mu}=\mu_{\text {vap }} / \mu_{\text {liq }}$ : the center of mass, falling velocity of mass center, and the circularity defined as a ratio of perimeter of circle to perimeter of droplet [5], and they are presented in Fig. 1-2. The droplet shapes when their center of mass pass $y=0.5$ are presented in Fig. 3. The reference case with $\lambda_{\rho}=0.1$ and $\lambda_{\mu}=0.1$ shows a droplet reaches the terminal velocity around $t=0.5 \mathrm{~s}$ while it has a stable ellipsoidal shape. Droplets with the same viscosity but lower density ratio, $\lambda_{\rho}=0.01$ and 0.001 , maintain circular shape and have linearly increasing velocity until they arrive at the bottom of domain. For the viscosity ratio study case, a droplet with higher viscosity ratio $\lambda_{\mu}=1.0$ develops a dimple at the top, while a droplet with lower viscosity ratio $\lambda_{\mu}=0.01$ has similar deformation rate with the reference droplet. The horizontally stretched shape of $\lambda_{\mu}=1.0$ leads to a drastic decrease of velocity after $0.4 \mathrm{~s}$ and lower terminal velocity than the reference case. 

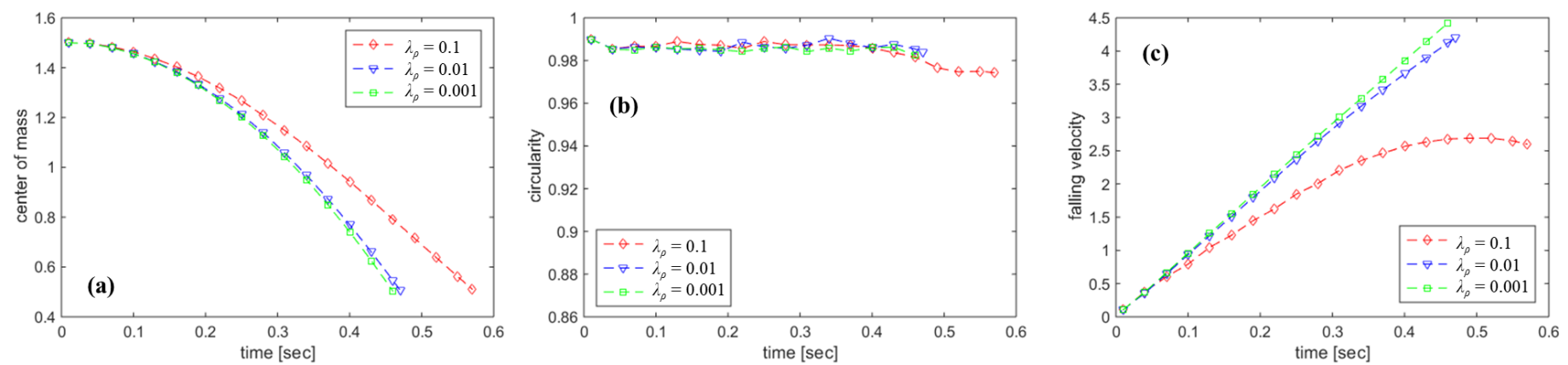

Fig. 1: (a) center of mass, (b) circularity, and (c) falling velocity depending on $\lambda_{\rho}$ with $\lambda_{\mu}=0.1$.
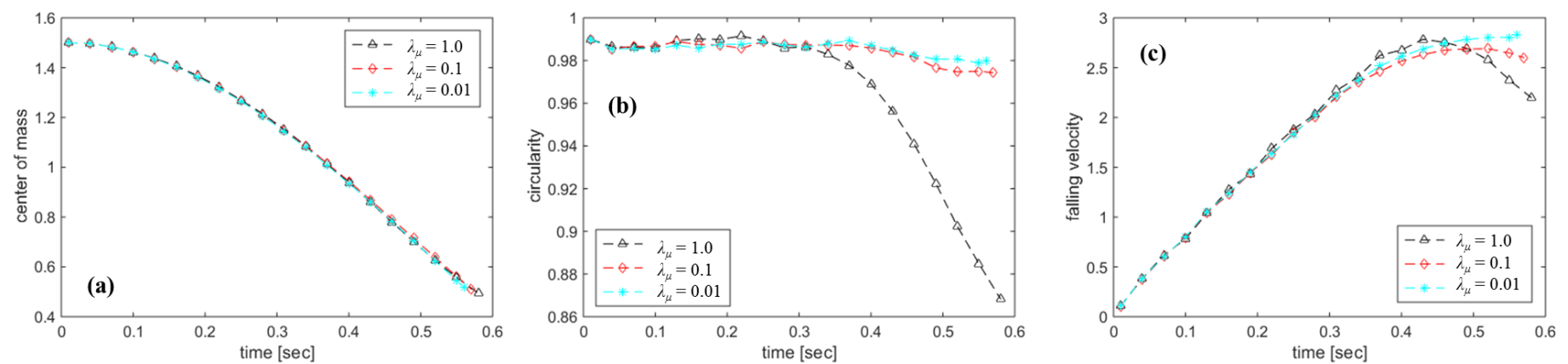

Fig. 2: (a) center of mass, (b) circularity, and (c) falling velocity depending on $\lambda_{\mu}$ with $\lambda_{\rho}=0.1$.
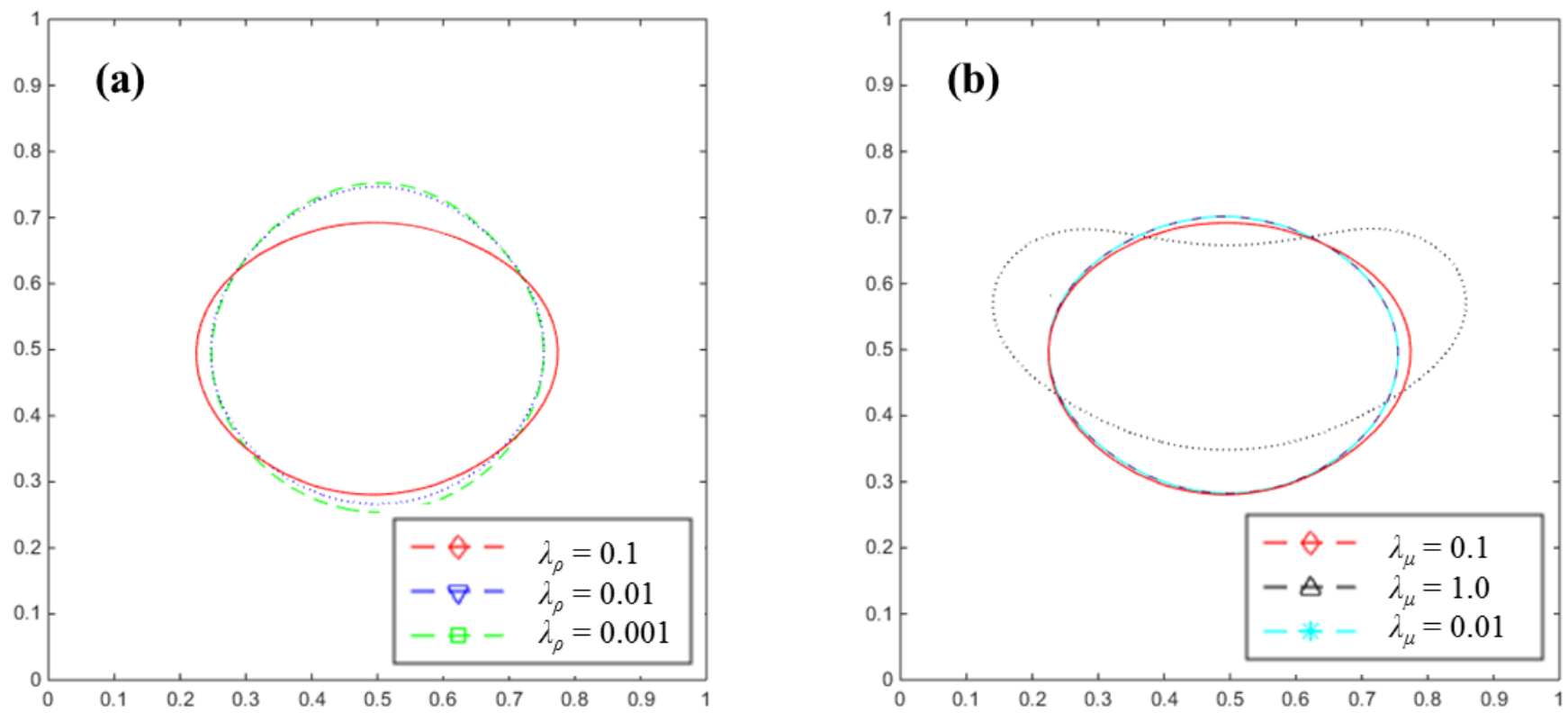

Fig. 3: Droplet shape when the center of mass is around $y=0.5$ (a) $\lambda_{\mu}=0.1$, (b) $\lambda_{\rho}=0.1$.

\section{References}

[1] J. Lowengrub and L. Truskinovsky, "Quasi-incompressible Cahn-Hilliard fluids and topological transitions," in Proceedings of the Royal Society of London A: Mathematical, Physical and Engineering Sciences, 1998, vol. 454, pp. 2617-2654. 
[2] F. Boyer, "A theoretical and numerical model for the study of incompressible mixture flows," Comput. Fluids, vol. 31, no. 1, pp. 41-68, 2002.

[3] H. Ding, P. D. Spelt, and C. Shu, "Diffuse interface model for incompressible two-phase flows with large density ratio," J. Computat. Phys., vol. 226, no. 2, pp. 2078-2095, 2007.

[4] J. Shen and X. Yang, "A phase-field model and its numerical approximation for two-phase incompressible flows and different densities and viscosities," SIAM J. Sci. Comput., vol. 32, no. 3, pp. 1159-1179, 2010.

[5] H. Abels, H. Garcke, and G. Grun, "Thermodynamically consistent, frame indifferent diffuse interface models for incompressible two-phase flows with different densities," Math. Mod. Meth. Appl. S., vol. 22, no. 3, 1150013, 2012. 